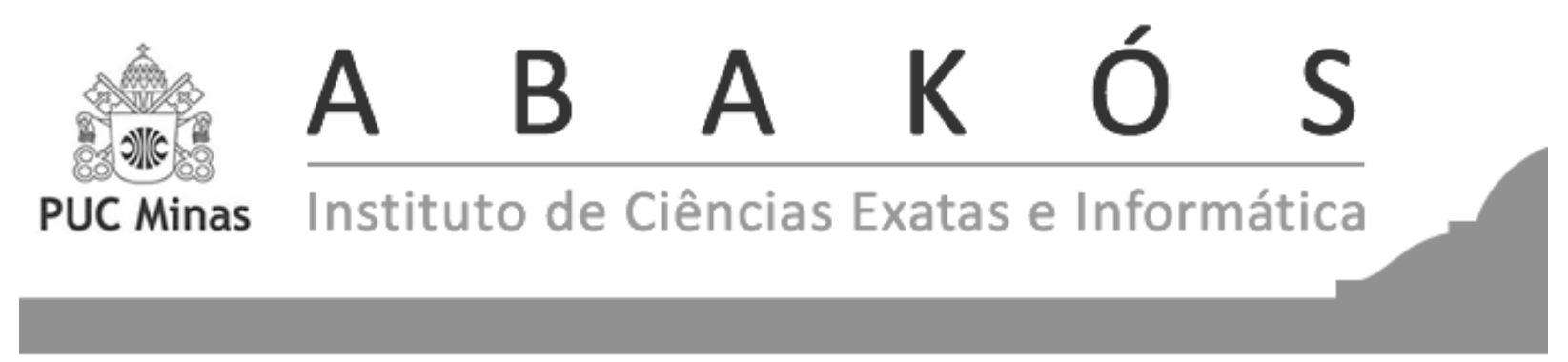

\title{
Ensino de Probabilidade e Estatística e Tecnologias da Informação e Comunicação: um cenário das produções brasileiras para os anos iniciais do Ensino Fundamental*
}

Teaching of Probability and Statistics and Information and Communication Technologies: scenario of Brazilian productions for the Elementary School

\author{
Cristiane de Fatima Budek Dias ${ }^{1}$ \\ Guataçara dos Santos Junior ${ }^{2}$
}

\begin{abstract}
Resumo
Este artigo traz um panorama das pesquisas realizadas no cenário brasileiro que têm como foco o ensino de Probabilidade e Estatística nos anos iniciais do Ensino Fundamental aliado às Tecnologias da Informação e Comunicação (TIC). Com o objetivo de verificar as discussões e as abordagens priorizadas nas pesquisas brasileiras, analisaram-se as produções disponibilizadas nas páginas dos programas de Mestrado (Acadêmico e Profissional) e Doutorado na área de Ensino, conforme tabela da CAPES dos cursos recomendados e reconhecidos. Para atender à proposta do presente artigo, realizou-se uma pesquisa exploratória, analisando-se de forma interpretativa as produções encontradas em nível nacional (20042016). Foram elencadas quatro categorias norteadoras: software utilizado nas atividades desenvolvidas; conceitos e conhecimentos estimulados; enfoques didáticos e metodológicos no uso das ferramentas; e perspectivas para a formação continuada de professores dos anos iniciais do Ensino Fundamental. Os resultados indicam que as tecnologias podem auxiliar o processo de ensino e aprendizagem dos conceitos referentes à Estatística, permitindo que a manipulação dos dados e os cálculos sejam facilitados. Entretanto, apontam também, para a necessidade de um maior número de pesquisas que abordem a temática, principalmente no que concerne à formação de professores.
\end{abstract}

Palavras-chave: Educação Estatística. Recursos tecnológicos. Ensino Fundamental.

\footnotetext{
* Submetido em 22/05/2017 - Aceito em 03/07/2018.

${ }^{1}$ Doutoranda em Ensino de Ciência e Tecnologia pela Universidade Tecnológica Federal do Paraná (UTFPR/Ponta Grossa) Bolsista Capes, Brasil-cristianed@alunos.utfpr.edu.br

${ }^{2}$ Professor do Programa de Pós-Graduação em Ensino de Ciência e Tecnologia Universidade Tecnológica Federal do Paraná (UTFPR/Ponta Grossa), Brasil-guata@utfpr.edu.br
} 


\begin{abstract}
This article presents an overview of the researches carried out in the Brazilian scenario that focus on Probability and Statistics in the initial years of Elementary Education combined with Information and Communication Technologies (ICT). In order to verify the discussions and approaches prioritized in Brazilian researches studies, the productions available in the pages of the Master's programs (Academic and Professional) and Doctorate in the Teaching area were studied, according to the CAPES table of recommended and recognized courses. To meet the proposal of this article, an exploratory research was carried out, analyzing the productions found (2004-2016) in an interpretative way. Four guiding categories were listed: software used in the activities developed; Concepts and knowledge stimulated; Didactic and methodological approaches in the use of tools; And perspectives for the continued formation of teachers of the initial years of Elementary School. The results indicate that the technologies can help the teaching and learning process of concepts related to statistics, allowing the manipulation of data and calculations to be facilitated. However, they also point to the need for a greater number of researches that approach the subject, especially about teacher training.
\end{abstract}

Keywords: Statistical Education. Technology resources. Elementary School. 


\section{INTRODUÇÃO}

As Tecnologias de Informação e Comunicação (TIC) estão presentes nas diversas práticas sociais, aprimorando a resolução de alguns problemas enfrentados pela humanidade, tornando mais eficazes os processos produtivos, estreitando relações e diminuindo o espaço geográfico entre as pessoas. Isso faz com que a inserção das TIC seja justificada e ampliada para os mais diferentes contextos, como no processo educativo.

Para Borba e Penteado (2012), a informática faz brotar transformações no interior do próprio conhecimento, repercutindo na prática pedagógica, na própria mídia e na percepção do conhecimento. Dessa maneira, entende-se que as mais diversas áreas são influenciadas pela tecnologia, assim como também podem influenciá-las. Os processos pedagógicos, então, não podem ficar alheios aos avanços da ciência e da tecnologia. Ensinar, nos dias de hoje, requer práticas diferenciadas e metodologias que considerem os estudantes do contexto atual, que estão imersos em um mundo permeado pelas tecnologias digitais (BORBA; PENTEADO, 2012).

Nesse sentido, entende-se que os conteúdos das mais diversas áreas do conhecimento podem ser abordados com apoio nas TIC, como é o caso dos conhecimentos relativos à Estatística. De acordo com Estevam e Kalinke (2013, p. 105) as TIC "podem caracterizar uma ferramenta de apoio poderosa ao ensino de Estatística, uma vez que suas características podem favorecer a tomada de consciência e compreensão de determinados conceitos estatísticos e probabilísticos". Para os autores, as tecnologias de informação e comunicação são recursos que possibilitam e tornam mais rápidas as investigações e a análise dos dados (ESTEVAM; KALINKE, 2013).

A facilitação na organização dos dados pelas ferramentas computacionais abre espaço para que professores e alunos possam analisar, interpretar e discutir as questões representadas nos gráficos e tabelas, ou seja, ganha-se tempo e espaço para a reflexão crítica e para possíveis tomadas de decisão a partir do que está exposto estatisticamente.

Entretanto, alguns aspectos precisam ser considerados, pois é importante que haja um planejamento adequado do professor e uma escolha correta das ferramentas. Também é importante que não se caia na ingenuidade de acreditar que as tecnologias sozinhas resolverão todos os problemas relacionados às dificuldades de aprendizagem dos conceitos estatísticos.

Compreende-se, então, que conhecer o que vem sendo proposto e tem sido pesquisado sobre o uso de ferramentas tecnológicas no ensino da Estatística é condição indispensável para que se verifiquem os resultados e as possíveis lacunas existentes, pois somente assim novos rumos poderão ser propostos.

Dessa maneira, este artigo traz um panorama das pesquisas realizadas no cenário brasileiro que têm como foco o ensino da Probabilidade e da Estatística nos anos iniciais do Ensino Fundamental aliado às TIC, com o objetivo de verificar as discussões e as abordagens priorizadas nas pesquisas brasileiras. 


\section{ENSINO DE PROBABILIDADE E ESTATATÍSTICA NOS ANOS INICIAIS DO EN- SINO FUNDAMENTAL}

Os conceitos que envolvem a Estatística passaram a fazer parte do currículo dos anos iniciais do Ensino Fundamental a partir do ano de 1997, quando foram propostos nos Parâmetros Curriculares Nacionais (PCN), no bloco de conteúdo denominado Tratamento da Informação do documento de Matemática. Essa inclusão é considerada um tanto tardia, visto que, no cenário internacional, tais conteúdos já se faziam presentes na educação da infância há, pelo menos, duas décadas.

Os PCN indicam que a Estatística tem "a finalidade de fazer com que o aluno venha a construir procedimentos para coletar, organizar, comunicar e interpretar dados, utilizando tabelas, gráficos e representações que aparecem frequentemente em seu dia a dia"(BRASIL, 1997, p. 56). Essas são habilidades fundamentais para o contexto do mundo atual, pois muitas estatísticas são apresentadas com frequência e precisam ser compreendidas pelo cidadão (BRASIL, 1997).

Há consenso entre pesquisadores e estudiosos de que a leitura e a interpretação das representações gráficas e tabulares são habilidades essenciais para o pleno exercício da cidadania (LOPES, 1998, 2008; CARVALHO, 2001; CAZORLA; CASTRO, 2008; PONTE; FONSECA, 2001; VIALI; SILVA, 2016). Isso porque o bombardeio de informações com dados é cada vez mais frequente no cotidiano das pessoas. Para além disso, é crucial que haja entendimento sobre a lógica dessas representações e da própria Estatística como fundamento para a tomada de decisão.

Instrumentalizar o cidadão com conhecimentos sobre como os dados são coletados, organizados e representados pode abrir caminho para questionamentos e para o pensamento crítico. E isso deve ser estimulado desde a infância. Na visão de Viali e Silva (2016, p. 4), tal prática vem "contribuir para que as crianças adquiram desde cedo a ideia de que a ciência é o melhor caminho e que é necessário sempre questionar todas as verdades e raciocinar por si. Assim se estará contribuindo para a formação de um cidadão crítico e com espírito científico". Os autores ainda destacam que, "quanto mais cedo tomarmos contato com os métodos e procedimentos de análise e interpretação de dados, mais natural e simples se tornará este processo"(VIALI; SILVA, 2016).

Então, tem-se por pressuposto que, embora ainda exista uma crença de que os conceitos probabilísticos e estatísticos são difíceis de serem compreendidos pelos estudantes dos anos iniciais, precisam se fazer presentes não apenas nas propostas curriculares, mas também, nas práticas docentes; ou seja, no contexto da sala de aula, possibilitando o desenvolvimento do pensamento estatístico.

Vale destacar que conhecimentos estatísticos são indispensáveis para o exercício de uma cidadania crítica, reflexiva e participativa (CARVALHO, 2001) e a criança, como cidadã, tem o direito à aprendizagem de tais conceitos para que construa seu pensamento crítico e possa participar ativamente da sociedade à qual pertence. 
Lopes (2008) afirma a necessidade de se tratar de conceitos de Estatística desde os anos iniciais, para que não se prive o estudante do entendimento mais amplo dos problemas sociais que ocorrem em sua realidade. É a partir desse entendimento que o estudante poderá refletir e fazer escolhas mais adequadas para o bem comum (LOPES, 2008).

Dada essa relevância da Estatística nos anos iniciais, observa-se que os PCN trazem como um dos objetivos da Matemática nessa etapa da Educação Básica:

Fazer observações sistemáticas de aspectos quantitativos e qualitativos do ponto de vista do conhecimento e estabelecer o maior número possível de relações entre eles, utilizando para isso o conhecimento matemático (aritmético, geométrico, métrico, algébrico, estatístico, combinatório, probabilístico); selecionar, organizar e produzir informações relevantes, para interpretá-las e avaliálas criticamente (BRASIL, 1997, p. 37).

Nesse sentido, o documento traz diversos conteúdos que precisam ser abordados no $1^{\circ} \mathrm{e}$ no $2^{\circ}$ ciclo dos anos iniciais. Tais conteúdos abrangem desde a leitura e a interpretação, como a própria coleta, a organização e a representação de dados. Há também destaque para a escrita de textos com base em dados. O que se pode afirmar é que, mesmo com uma abordagem superficial e sem instruções mais claras ao professor, a Estatística está presente no currículo e traz indícios da necessidade do trabalho partindo-se da coleta de dados, aspecto primordial quando se trata do processo de ensino e aprendizagem dos conceitos estatísticos.

Autores como Silva (2013), Grando, Nacarato e Lopes (2014) e Santos e Ponte (2013) afirmam que é imprescindível que o trabalho com a estatística seja firmado a partir de investigações estatísticas, ou seja, por meio da pesquisa e da coleta dos dados. Também é importante destacar a relevância da escrita, pois isso estimula a argumentação e o uso de terminologias próprias da área (SILVA, 2013; GRANDO et al., 2014; SANTOS; PONTE, 2013).

Alguns autores, ainda, acreditam que o ensino de Estatística, aliado à tecnologia, traz facilidades para que o aluno possa compreender a lógica das representações dos dados e, também, um ganho significativo de tempo, que poderá ser melhor aproveitado com reflexões sobre as informações coletadas e acerca de possíveis ações diante das conclusões conseguidas (BATANERO; DÍAZ, 2013; LIRA; MONTEIRO, 2011; BEN-ZVI, 2011).

Essas são propostas a serem consideradas quando se trata da necessidade de educar estatisticamente as crianças e de propor a elas vivências de suas realidades. Em outras palavras, são práticas consideradas adequadas para a efetivação da Educação Estatística.

\subsection{Ensino de Estatística e Tecnologias da Informação e Comunicação}

Acredita-se que as TIC podem possibilitar que os conceitos probabilísticos e estatísticos sejam assimilados com mais facilidade pelos alunos e, também, que inúmeros dados e investigações possam ser representados com mais clareza, evitando um trabalho cansativo.

Para Batanero (2001), o dinamismo, a velocidade e o aumento gradativo de novos aplicativos de software, que possibilitam a exploração de todas as dimensões dos processos esta- 
tísticos, como o planejamento amostral, o delineamento da coleta e a manipulação dos dados, as análises, as interpretações e a comunicação dos resultados, demonstram a pertinência do uso das TIC no trabalho com a Probabilidade e Estatística. Essas ferramentas, ainda, possibilitam que novos tópicos sejam integrados ao ensino de Estatística (BATANERO, 2001).

Ben-Zvi (2011), ao refletir sobre os elementos-chave para o ambiente de aprendizagem para o raciocínio estatístico (Statistical Reasoning Learning Environment - SRLE), revela que um desses elementos é a integração de ferramentas tecnológicas no ensino. Para o autor, existe uma gama de ferramentas tecnológicas que podem auxiliar no desenvolvimento do raciocínio e da compreensão dos estudantes sobre a Estatística, como computadores, calculadoras gráficas, Internet, software estatístico e aplicativos da Web (BEN-ZVI, 2011).

Nessa perspectiva, o centro do processo de ensino e aprendizagem de Estatística passa a ser o entendimento sobre como escolher métodos de análise mais adequados e como interpretar os resultados. Essas ferramentas não se concentram somente na geração de representações estatísticas ou na análise de dados, mas proporcionam a visualização de conceitos e auxiliam no desenvolvimento da compreensão de ideias abstratas por meio das simulações (BEN-ZVI, 2011).

Nessa mesma linha de pensamento, Ponte e Fonseca (2001), ao discutirem sobre as propostas curriculares de três países (Portugal, Inglaterra e Estados Unidos) afirmam que as TIC apresentam influências fundamentais no processo de ensino de Estatística,

[...] possibilitando a realização de todo o tipo de cálculos e facilitando o uso de uma grande variedade de formas de representação. Deste modo, as TIC possibilitam o tratamento de dados reais, em vez do tradicional trabalho com amostras de pequena dimensão onde os valores são escolhidos de modo artificial para proporcionarem cálculos simples. Mais recentemente, a Internet, onde é possível obter uma imensa variedade de dados estatísticos, surgiu como um recurso de grande alcance para o ensino-aprendizagem deste tema (PONTE; FONSECA, 2001, p. 5).

Os autores ainda destacam que, no que se refere às TIC, os documentos dos três países analisados aconselham sua utilização para a representação de dados e a realização de cálculos. Porém, o documento americano vai além, "ao valorizar a utilidade das TIC na investigação dos efeitos da mudança de alguns dados numa amostra, no uso de simulações probabilísticas e na realização de pesquisas na Internet"(PONTE; FONSECA, 2001, p. 22).

Conforme Batanero e Diaz (2013), a tecnologia possibilita a aplicação da Estatística de forma mais facilitada, com maior evidência nas atividades de interpretação que em cálculos rotineiros. Assim, ao fazer uso da tecnologia no ensino de Estatística, o professor estará possibilitando a seus alunos tempo e espaço para reflexões mais abrangentes sobre a realidade investigada. Tal fato pode levar à superação da visão determinística com que, frequentemente, é tratada a Probabilidade e Estatística dentro da sala de aula (BATANERO; DÍAZ, 2013).

Lira e Monteiro (2011) também evidenciam esse aspecto em seu estudo sobre a utilização de um software no ensino de Estatística, apontando que há um melhor aproveitamento do tempo e, consequentemente, traz ganhos para o processo interpretativo das informações obtidas 
nas investigações realizadas pela turma. No uso de aplicativos de software que possibilitem o trabalho com gráficos, o professor pode elaborar atividades que favoreçam a leitura, a interpretação, a interdisciplinaridade e a constatação da importância da Matemática, na resolução de problemas da vida cotidiana (LIRA; MONTEIRO, 2008).

Ao permitir o desenvolvimento dessas habilidades de interpretação por meio do uso do computador "antes do ensino explícito de convenções e tecnicismos para desenhar gráficos, pode-se provocar uma mudança na 'lógica' curricular que hierarquiza os tipos de gráficos, do mais fácil (barras) para o mais difícil (linhas, dispersão)"(LIRA; MONTEIRO, 2008).

Nesse sentido, pode-se dizer que a tabulação dos dados e a representação dos mesmos em gráficos não constitui o fim da investigação estatística, pois, abre-se espaço para uma reflexão crítica dos dados levantados e, também, para a superação da hierarquia e da linearidade com que as representações gráficas e tabulares são propostas no currículo escolar.

Segundo Estevam e Kalinke,

[...] os recursos tecnológicos podem ampliar os limites do ensino de Estatística e possibilitam simulações e explorações que favorecem a compreensão de conceitos e habilidades que envolvem o processo de análise de dados, em detrimento de aspectos algébricos e/ou puramente estruturais (ESTEVAM; KALINKE, 2013, p. 104).

Nessa perspectiva, subentende-se que o uso de recursos didáticos tecnológicos pode favorecer a elaboração de problemas e de questões investigativas, nas quais as respostas matemáticas podem ser exploradas de forma menos cristalizada e sem a ênfase apenas em cálculos.

Martins (2013) assinala que o uso das tecnologias associadas à Estatística vem se justificando, tanto pela relevância dos próprios professores estarem a par dos avanços da sociedade e dos conteúdos correlatos a essa área, bem como, pelas oportunidades de aprendizagem promovidas com os recursos tecnológicos. Compreender as transformações sociais e as necessidades da sociedade atual pode ser um passo importante para que os professores se desvinculem de práticas tradicionais de ensino, principalmente quando se trata da Probabilidade e Estatística. Acredita-se que é condição indispensável, portanto, que as características de aprendizagem dos estudantes de hoje sejam consideradas (MARTINS, 2013).

Batanero (2001) aponta para a existência de formas diversas no uso do computador nas práticas didático-pedagógicas, refletindo que todas são de grande valia e representam uma revolução no processo de ensino e aprendizagem. Assim, considera-se que, por meio do trabalho com as TIC, pode ser possível uma aproximação com a realidade, coletando, organizando e analisando-se informações reais que possam trazer reflexões mais críticas a respeito do que se vivencia em muitos dos aspectos sociais, políticos, econômicos e culturais. A ideia de um trabalho fictício apenas para que se cumpra o currículo pode ser superada quando se apoia em recursos tecnológicos que facilitam cálculos e permitem o acesso às informações em um curto espaço de tempo (BATANERO, 2001).

A Estatística proporciona atividades interessantes para a introdução dos estudantes no uso dos recursos informáticos mais habituais, como os editores de texto e as planilhas eletrônicas, assim como no desenvolvimento das habilidades para uso de calculadoras científicas e 
gráficas (BATANERO, 2001), constituindo-se num passo importante para abarcar, no ensino de Probabilidade e Estatística, o desenvolvimento de outras habilidades e competências que contribuirão para uma formação mais completa dos estudantes.

Nessa perspectiva, percebe-se que o uso de recursos tecnológicos e outros materiais didáticos, na prática pedagógica, pressupõe ao professor muito mais do que a busca por uma aula agradável ao aluno, mas o constante desafio de tornar a sala de aula um ambiente de motivação, de reflexão e de construção do conhecimento. No contexto em que se apresentam os recursos tecnológicos dos dias de hoje, entende-se que as ferramentas para o ensino de Probabilidade e Estatística, precisam levar os alunos a avaliar as informações que lhe são oferecidas, tornandoos críticos.

\section{PROCEDIMENTOS METODOLÓGICOS}

Esta pesquisa é de natureza exploratória e descritiva, sendo que, para sua concretização, foi realizado, primeiramente, o levantamento dos programas de pós-graduação nacionais (Mestrados e Doutorados) recomendados pela CAPES na área de Ensino. Posteriormente, uma pesquisa nas páginas desses programas, compreendendo o período de 2004 a 2016 Foram selecionadas as produções que traziam a Estatística como objeto de pesquisa e foram utilizadas as palavras-chave: estatística, estocástica, probabilidade, análise combinatória, tratamento da informação, média, moda e mediana. Foram encontrados 161 trabalhos.

Num segundo momento, houve a seleção dos trabalhos voltados ao ensino de Estatística para os anos iniciais do Ensino Fundamental, sendo que, dos 161 encontrados, 23 enquadram-se nessa categoria. Desses, 17 são de Mestrado Acadêmico; 6 de Mestrado Profissional e 2 são Teses de Doutorado. A partir dessa seleção, separou-se as produções que tinham como foco o ensino de Estatística mediado por recursos tecnológicos. Assim, verificou-se a existência de 7 dissertações, sendo 5 de Mestrado Acadêmico e 2 de Mestrado Profissional. Não foi encontrado nenhum trabalho de Doutorado que tratasse do tema, conforme se verifica no Quadro 01. 
Quadro 1 - Dissertações selecionadas para a análise neste trabalho

\begin{tabular}{|c|c|c|c|c|}
\hline Instituição & Ano & Título & Autor & Nível \\
\hline PUC/SP & 2005 & $\begin{array}{l}\text { Introduzindo o conceito de } \\
\text { Média Aritmética na } 4^{\mathrm{a}} \text { série } \\
\text { do ensino fundamental } \\
\text { usando o ambiente } \\
\text { computacional }\end{array}$ & $\begin{array}{l}\text { Rosana Catarina } \\
\text { Rodrigues de } \\
\text { Lima }\end{array}$ & $\begin{array}{l}\text { Mestrado } \\
\text { Acadêmico }\end{array}$ \\
\hline URI/RS & 2011 & $\begin{array}{l}\text { Desenvolvimento de um } \\
\text { sistema modelo para ensino } \\
\text { aprendizagem de estatística } \\
\text { nas séries iniciais }\end{array}$ & $\begin{array}{l}\text { Luciana } \\
\text { Rockenbach de } \\
\text { Moraes }\end{array}$ & $\begin{array}{l}\text { Mestrado } \\
\text { Profissional }\end{array}$ \\
\hline UFPE & 2011 & $\begin{array}{l}\text { A interpretação de gráficos } \\
\text { em um ambiente } \\
\text { computacional por alunos de } \\
\text { uma escola rural do } \\
\text { município de Caruaru-PE }\end{array}$ & $\begin{array}{l}\text { Iane Maria } \\
\text { Pereira Alves }\end{array}$ & $\begin{array}{l}\text { Mestrado } \\
\text { Acadêmico }\end{array}$ \\
\hline UFPE & 2011 & $\begin{array}{l}\text { O uso do Tinkerplots para } \\
\text { exploração de dados por } \\
\text { professores de escolas rurais }\end{array}$ & $\begin{array}{l}\text { Andreika } \\
\text { Asseker } \\
\text { Amarante }\end{array}$ & $\begin{array}{l}\text { Mestrado } \\
\text { Acadêmico }\end{array}$ \\
\hline UFPE & 2013 & $\begin{array}{l}\text { Explorações sobre média no } \\
\text { software Tinkerplots } 2.0 \text { por } \\
\text { estudantes do } \\
\text { Fundamental }\end{array}$ & $\begin{array}{l}\text { Robson da Silva } \\
\text { Eugênio }\end{array}$ & $\begin{array}{l}\text { Mestrado } \\
\text { Acadêmico }\end{array}$ \\
\hline UFPE & 2014 & $\begin{array}{l}\text { Software educativo } \\
\text { Tinkerplots } 2.0 \text { : } \\
\text { possibilidades e limites para } \\
\text { a interpretação de gráficos } \\
\text { por estudantes do Ensino } \\
\text { Fundamental }\end{array}$ & $\begin{array}{l}\text { Siquele Roseane } \\
\text { de Carvalho } \\
\text { Campêlo }\end{array}$ & $\begin{array}{l}\text { Mestrado } \\
\text { Acadêmico }\end{array}$ \\
\hline UTFPR & 2016 & $\begin{array}{l}\text { Ambiente Virtual de } \\
\text { Aprendizagem para o ensino } \\
\text { de probabilidade e estatística } \\
\text { nos anos iniciais do Ensino } \\
\text { Fundamental }\end{array}$ & $\begin{array}{l}\text { Cristiane de } \\
\text { Fátima Budek } \\
\text { Dias }\end{array}$ & $\begin{array}{l}\text { Mestrado } \\
\text { Profissional }\end{array}$ \\
\hline
\end{tabular}

Fonte: Dados da Pesquisa (2016).

Discriminadas as produções a serem analisadas neste estudo, realizou-se o download dos trabalhos, efetuando-se a leitura dos resumos com atenção para pontos como: objetivos, problema, participantes da pesquisa, recurso tecnológico utilizado e resultados. Em alguns casos, houve a necessidade da realização de uma leitura mais aprofundada, uma vez que alguns trabalhos não explicitavam com clareza os recursos utilizados e o trabalho proposto no início.

A presente análise interpretativa está subdividida em quatro categorias norteadoras:

I - Software utilizado nas atividades desenvolvidas: nessa categoria, são discutidas as ferramentas utilizadas e/ou desenvolvidas nas pesquisas analisadas; 
II - Conceitos e conhecimentos estimulados: essa categoria aponta os principais conceitos e conhecimentos estatísticos estimulados a partir das práticas realizadas nas pesquisas analisadas;

III - Enfoques didáticos e metodológicos no uso das ferramentas: nessa categoria, analisouse os principais enfoques didáticos metodológicos dos pesquisadores ao fazerem uso do recurso tecnológico com propósitos de ensino e aprendizagem dos conceitos estatísticos;

\section{IV - Perspectivas para a formação continuada de professores dos anos iniciais do Ensino}

Fundamental: essa categoria trata das pesquisas que apresentam perspectivas para a formação de professores, ou seja, daquelas desenvolvidas, especificamente, com grupos de professores atuantes nos anos iniciais do Ensino Fundamental.

\section{ANÁLISE DOS RESULTADOS}

De acordo com o tema tratado neste trabalho, grande parte das pesquisas avaliadas envolveu atividades relacionadas a conceitos estatísticos utilizando-se aplicativos de software, em sua maior parte, específicos de Estatística ou educativos.

Verifica-se que 4 das produções utilizaram o TinkerPlots: Alves (2011); Amarante (2011); Eugênio (2013) e Campêlo (2014), todas da Universidade Federal de Pernambuco (UFPE). Uma pesquisa foi desenvolvida com o Tabletop, Lima (2005), e duas, Moraes (2011) e Dias (2016), tendo como base o desenvolvimento de sistemas e ambientes virtuais para o ensino de estatística. Moraes (2011) denomina seu produto de Sistema Estatístico para Séries Iniciais (SESINI). O ambiente virtual desenvolvido por Dias (2016) tem a denominação de Stat1 (ALVES, 2011; AMARANTE, 2011; EUGÊNIO, 2013; CAMPÊLO, 2014; LIMA, 2005; MORAES, 2011; DIAS, 2016).

O TinkerPlots é um software educacional comercial de análise de dados, desenvolvido por Konold \& Miller (2001) na Universidade de Massachusetts, centrando-se no trabalho com crianças e jovens, abrangendo estudantes na faixa etária dos anos iniciais do Ensino Fundamental brasileiro. Segundo seus autores, a ferramenta consiste num complexo instrumento de ensino de Estatística que motiva o aluno a realizar atividades dentro da sala de aula e fora dela (ALVES, 2011).

O TinkerPlots possui um espaço dinâmico e interativo, que permite a organização e a exploração de diferentes representações gráficas de dados. A tela inicial é formada por uma área em branco e o idioma é o inglês, língua na qual são apresentadas suas ferramentas básicas: Cards, Table, Plot, Slider e Text.

A ferramenta Cards permite o registro e a criação do banco de dados de uma determinada pesquisa. A ferramenta Table distribui os dados em forma de tabela. Já a ferramenta Plot permite a manipulação e a construção de várias representações dos dados e o cruzamento de variáveis, agregando mais alguns recursos, como, por exemplo, Separate, Order, Stack, Averages, entre outas. Ao ativar a ferramenta Plot, um menu com novos recursos é habilitado para o 
trabalho do usuário com os plots. Slider é a ferramenta que possibilita a realização de alterações na amostra dos dados a serem trabalhados. A ferramenta Text disponibiliza na tela uma caixa de texto, na qual, podem ser editadas informações sobre a tarefa em que se está trabalhando. As ferramentas Cards e Table estão interligadas, apresentando o mesmo banco de dados de formas diferentes (ALVES, 2011; AMARANTE, 2011; EUGÊNIO, 2013).

No estudo de Eugênio (2013), há enfoque na ferramenta Averages, parte dos recursos da função Plot. Essa ferramenta, quando acionada, representa simbólica e numericamente as medidas de tendência central e, como seu foco está voltado para o trabalho com a média aritmética, é amplamente explorada com os alunos. De acordo com o pesquisador, o TinkerPlots disponibiliza inúmeros caminhos para que o estudante possa chegar a conclusões sobre os a dados (EUGÊNIO, 2013).

De acordo com Campêlo (2014, p. 42), o TinkerPlots possibilita que os estudantes "percorram e estabeleçam diversas relações entre os diferentes momentos do tratamento de dados tais como: a coleta, organização, formulação e teste de hipóteses sobre os dados". Também permite o cruzamento de variáveis (CAMPÊLO, 2014, p. 42).

As ferramentas possibilitam a criação de gráficos e tabelas a partir de bancos de dados. Há, também, a possibilidade da produção de relatórios com gráficos, textos e ilustrações sobre esses dados. Portanto, o software pode ser uma importante ferramenta para a ampliação das experiências com o saber matemático, ajudando estudantes a desenvolver o raciocínio estatístico e a aprender novas formas de representar dados (CAMPÊLO, 2014).

Alves (2011) e Amarante (2011) evidenciam a relevância das ferramentas Separate e Gradiente da função Plot, no trabalho de interpretação dos gráficos pelos participantes da pesquisa. De acordo com Amarante (2011), essas ferramentas oportunizaram a percepção dos valores ordenados na escala, auxiliando no processo de interpretação dos dados (ALVES, 2011; AMARANTE, 2011).

Outro recurso comercial utilizado em uma das produções analisadas nesta pesquisa é o Tabletop, que consiste em um "pacote estatístico", com finalidade educativa. O software é destinado à organização e à manipulação de dados, permitindo a inserção de etapas de construção, exploração e análise de determinado banco de dados.

É permitida a criação de três tipos de gráficos: o diagrama de Venn; o gráfico de frequência e o gráfico de dupla entrada e é possível mudar o tipo de variável para gerar um novo gráfico. A disposição dos dados na tabela exibe-os de "forma bruta e não simplificada", o que a autora considera como uma vantagem sobre os livros. Para a pesquisadora, o fato dos dados organizados na representação tabular poderem ser tratados e visualizados de diferentes formas, torna o Tabletop um ambiente propício para a aprendizagem (LIMA, 2005).

O Tabletop se justifica no trabalho de Lima (2005) pela sua adequação à faixa etária das crianças, às suas características técnico-pedagógicas e à sua flexibilização na construção e reconstrução de gráficos, "esta facilidade poderá encorajar os alunos a investigarem qual dos gráficos oferecerá melhor a informação desejada, ou mesmo, buscar uma representação esteticamente mais agradável"(LIMA, 2005, p. 66). 
Moraes (2011) desenvolve o SESINI, que consiste em um software para a resolução de problemas e atividades relacionadas à construção de gráficos pictóricos, que podem ser construídos a partir de figuras cadastradas, tanto pelo professor, como pelos alunos. Para ter acesso ao sistema, é necessário um cadastro prévio, sendo que, de acordo com a descrição do usuário (professor/aluno), há o redirecionamento às telas correspondentes a cada um (MORAES, 2011).

A validação do sistema ocorreu com discentes de um curso de Pedagogia e, segundo a pesquisadora, "pode-se afirmar que o mesmo foi de grande aceitação entre os mesmos"(MORAES, 2011, p.72). Conforme suas colocações, esses estudantes apontam que a ferramenta traz facilidade ao aprendizado dos alunos. Não foi possível encontrar o SESINI para que mais detalhes pudessem ser apresentados.

Dias (2016) é a segunda pesquisadora que trabalha no desenvolvimento de um software específico para o ensino de Estatística nos anos iniciais. O Ambiente Virtual de Aprendizagem (AVA) foi desenvolvido tendo como aporte os documentos curriculares oficiais e as práticas docentes. A ferramenta utilizada para a construção do AVA foi o Java Enterprise Edition 7 (JEE 7), e o ambiente pode ser acessado em multiplataformas (DIAS, 2016).

O AVA possui ferramentas que trazem "conceitos, espaços para a edição de informações, para a realização de pesquisas, para a formação continuada dos professores e para a interatividade entre colegas de profissão"(DIAS, 2016, p. 122). Algumas das ferramentas são abertas e permitem acesso para qualquer pessoa. Já outras são restritas a usuários cadastrados como professores e/ou alunos. Há locais específicos para cada perfil de usuário. Os professores são os responsáveis pelo próprio cadastro e pelo cadastro de suas turmas e alunos. A partir disso, esses usuários podem interagir na ferramenta, elaborando questionários de pesquisas, respondendo e gerando dados e representações gráficas dessas pesquisas. Também podem publicar esses resultados em forma de texto em um espaço denominado de Blog.

Segundo Dias (2016, p.122), tal "instrumento foi desenvolvido de forma que suas ferramentas se constituíssem em espaços para a interação entre professores e alunos; alunos e alunos; professores e professores; entre escolas diferentes; e entre escola e sociedade"(DIAS, 2016).

Observa-se que a pesquisadora desenvolveu seu software em conjunto com professores atuantes nos anos iniciais do Ensino Fundamental, a partir da análise das práticas e da participação ativa desses professores no momento de estruturar cada uma das ferramentas presentes no ambiente. Os professores foram convidados a participar no desenvolvimento propriamente dito, e não apenas na validação do instrumento já pronto, processo ao qual a autora delega bastante importância, pois, "a participação dos professores no processo de desenvolvimento de recursos tecnológicos pode ser fundamental para que esses recursos sejam, realmente, desenvolvidos com atenção aos anseios dos professores e à realidade da sala de aula"(DIAS, 2016, p. 133). 


\subsection{Conceitos e Conhecimentos Estimulados}

A Estatística é tratada nos anos iniciais do Ensino Fundamental dentro do bloco Tratamento da Informação e compreende os conceitos estatísticos, probabilísticos e combinatórios. Nessa etapa da Educação Básica, os alunos devem ter contato com a coleta, a organização, a leitura e a interpretação de dados (BRASIL, 1997). São discutidas, nessa categoria, as produções que evidenciam os conceitos e conhecimentos estatísticos estimulados durante a realização da pesquisa.

Duas das produções levantadas trabalham os conceitos referentes às Medidas de Tendência Central, focando-se na introdução e na exploração dos conceitos de média aritmética: Lima (2005) e Eugênio (2014) (LIMA, 2005; EUGÊNIO, 2013).

Lima (2005) investigou a introdução do conceito de média aritmética com base no uso das representações gráficas e com o auxílio do ambiente computacional. Abordou os conceitos de média aritmética e estimulou o desenvolvimento da leitura e interpretação de gráficos, partindo de problemas do cotidiano dos alunos (LIMA, 2005).

A autora afirma que o contato com o conceito de média aritmética na $4^{\mathrm{a}}$ série do Ensino Fundamental (turma em que realizou sua pesquisa) é capaz de possibilitar ao estudante um entendimento mais efetivo desse conceito, já que o mesmo será retomado posteriormente nas séries seguintes, articulando-se às demais medidas de tendência central. $\mathrm{O}$ estudo tem a média como seu foco pela sua frequente utilização nos meios de comunicação e por ser um conceito importante na análise de dados e na tomada de decisão (LIMA, 2005).

Da mesma forma, Eugênio (2013) analisa as explorações sobre a média por estudantes do $5^{\circ}$ e do $9^{\circ}$ ano do Ensino Fundamental. O pesquisador, em suas conclusões, afirma que esse trabalho associado à construção e à interpretação de gráficos podem ser inseridos na educação escolarizada a partir do $5^{\circ}$ ano (EUGÊNIO, 2013).

Percebe-se que ambos partem da leitura e da interpretação de gráficos para a abordagem do conceito de média aritmética e, em seus resultados, apontam que esse tipo de tratamento favoreceu o entendimento dos alunos.

Amarante (2011) investigou o uso do TinkerPlots para a exploração de dados bivariados, propondo cruzamento entre as variáveis quantitativas, as variáveis qualitativas e uma variável quantitativa e uma qualitativa (AMARANTE, 2011).

O processo de leitura e interpretação de gráficos é analisado nas pesquisas de Alves (2011) e Campêlo (2014). Os resultados das pesquisas revelaram que a possibilidade de diferentes representações para um conjunto de dados facilitou o processo de exploração, a compreensão e a atribuição de significado às representações (ALVES, 2011; CAMPÊLO, 2014).

Os resultados da pesquisa apontam para a importância do software no favorecimento de diferentes formas de representação para os dados e o enriquecimento de estratégias de resolução de problemas envolvendo o Tratamento da Informação. A partir da análise baseada nos critérios considerados, pudemos constatar a adequação do software TinkerPlots para a exploração de problemas envolvendo interpretação e produção de gráficos estatísticos, bem como a promoção 
de situações envolvendo organização e análise de dados (CAMPÊLO, 2014).

Campêlo (2014) aponta a utilização de várias atividades envolvendo uma variável; duas variáveis; análise de tendência do gráfico, e construção de um novo caso. Em Alves (2011), as questões exploraram a correlação de variáveis (CAMPÊLO, 2014; ALVES, 2011).

O sistema desenvolvido por Moraes (2011), trabalha com a construção de gráficos pictóricos e pesquisa de Dias (2016) aponta para o trabalho com diferentes conceitos e conhecimentos estatísticos, de acordo com os conteúdos elencados nos PCN (BRASIL, 1997) e nas Diretrizes Curriculares Municipais do município onde foi desenvolvida a pesquisa (MORAES, 2011; DIAS, 2016; BRASIL, 1997).

Descritos os conceitos abordados e os conhecimentos estimulados, apresenta-se a seguir os enfoques didáticos e metodológicos no uso das ferramentas tecnológicas para o ensino desses conceitos nas pesquisas analisadas.

\subsection{Enfoques didáticos e metodológicos no uso das ferramentas}

Assim como a escolha correta da ferramenta tecnológica é fundamental para que os objetivos de ensino e aprendizagem sejam alcançados, é igualmente importante que os pressupostos metodológicos e didáticos sejam coerentes com esses objetivos. Conforme apontam, Lira e Monteiro (2011, p. 765) introduzir computadores na escola não significa garantir êxito no ensino de Estatística e Matemática, pois, é necessário atenção às práticas pedagógicas e às ferramentas utilizadas. Em outras palavras, o professor precisa saber qual instrumento utilizar e como abordar os conceitos estatísticos tendo como base o recurso escolhido (LIRA; MONTEIRO, 2011, p. 765).

Nesse sentido, pode-se verificar, nas produções analisadas, que algumas assentaram suas propostas na Teoria dos Campos Conceituais proposta por Vergnaud (1982). Tal teoria, tem como premissa que o conhecimento se organiza em campos conceituais, que requerem um largo espaço de tempo para serem dominados pelo indivíduo (VERGNAUD, 1982). As pesquisas de Lima (2005) e Eugênio (2013), baseiam-se nessa teoria (LIMA, 2005; EUGÊNIO, 2013).

Algumas produções, também, aportam-se nos níveis de compreensão de gráficos: ler os dados, ler entre os dados e ler além dos dados para elaborar suas atividades, como é o caso de Lima (2005). O primeiro nível é a leitura literal dos dados (título, rótulos, eixos), sem interpretação da informação, o segundo é a leitura entre os dados, que requer comparações e uso de conceitos matemáticos e, o terceiro nível, a leitura além dos dados, que pressupõe fazer inferências e previsões, o que requer conhecimento prévio do estudante (LIMA, 2005; CURCIO, 1981).

Duas produções consideram as propriedades da média aritmética, Lima (2005) e Eugênio (2013). As propriedades foram consideradas de acordo com Strauss e Bichler (1988): a) a média está localizada em valores extremos; b) as somas das variações da média é zero; c) a média é influenciada por valores diferentes da média; d) a média não é necessariamente igual a 
um dos valores que está sendo somado; e) a média pode ser uma fração sem equivalência na realidade física; f) quando se calcula a média, um valor zero, se aparecer, deve ser considerado; g) o valor médio é representativo dos valores, cuja média foi calculada (LIMA, 2005; EUGÊNIO, 2013; STRAUSS; BICHLER, 1988).

Alves (2011), Amarante (2011), Eugênio (2013) e Campêlo (2014) partem da resolução de problemas para estimular a aprendizagem dos conceitos estatísticos. Fazem uso do próprio banco de dados do TinkerPlots para compor suas atividades. Os contextos de uso e questões foram adaptados e traduzidos para a utilização na pesquisa (EUGÊNIO, 2013; CAMPÊLO, 2014). Em todos esses estudos, aos participantes da pesquisa é, inicialmente, proposta uma etapa de familiarização com o software, seguida de uma etapa de interpretação de problemas envolvendo bancos de dados e a construção de diferentes visualizações (ALVES, 2011; AMARANTE, 2011).

Amarante (2011, p. 146) aponta que as questões utilizadas em seu estudo requeriam a percepção da relação entre variáveis, atribuindo a essa tarefa um sentido desafiador, "pois, para interpretar os dados, era necessário testar hipóteses e observar os indícios que podiam ser utilizados para justificar a resposta construída”. A autora menciona que os professores envolvidos na pesquisa construíram diferentes tipos de representações por meio das ferramentas do TinkerPlots, aspecto que auxiliou na interpretação dos dados (AMARANTE, 2011, p.146).

Apenas as pesquisas de Lima (2005) e Alves (2011) apresentam processos de investigação para a abordagem dos conceitos estatísticos. Lima (2005) realiza a coleta direta com os alunos, partindo para a organização e a construção de tabelas e gráficos no Tabletop. Alves (2011) também realiza, em uma das primeiras etapas de seu estudo, a coleta de dados a partir de uma investigação com os alunos, mas, esses são utilizados apenas na etapa de conhecimento do software. Vale, aqui, reiterar a importância dos processos de investigação para o ensino de Estatística, como apontado por diversos autores, entre eles, Silva (2013), Grando, Nacarato e Lopes (2014), Santos e Ponte (2013) (LIMA, 2005; ALVES, 2011; SILVA, 2013; GRANDO et al., 2014; SANTOS; PONTE, 2013).

A pesquisa de Moraes (2011) não apresenta detalhes sobre o enfoque didático e metodológico, mas aponta que seu sistema destaca a resolução de problemas na construção dos gráficos. Em Dias (2016), observa-se uma proposta pautada em teorias que versam sobre os conhecimentos básicos para o professor, baseando-se em Shulman (1986, 2005), Mishra e Koehler (2006, 2008), Nóvoa (2009, 2013). O AVA desenvolvido tem características que visam a investigação como proposta para o ensino de Estatística e destaca a comunicação nas propostas de publicação das pesquisas realizadas pelos alunos e professores (MORAES, 2011; DIAS, 2016). 


\subsection{Panoramas para a formação continuada de professores dos anos iniciais do Ensino Fundamental}

Tem-se por pressuposto que a formação continuada de professores é um aspecto fundamental tanto para o ensino da Estatística como para a incorporação efetiva das tecnologias no processo educativo.

Em seus estudos sobre uso de simuladores e da tecnologia no ensino da estocástica, Souza e Lopes (2011) evidenciam a importância das intervenções do professor durante o processo de ensino com uma ferramenta computacional. Também, apontam para a relevância de um bom planejamento para o estímulo do aluno. Entretanto, sabe-se que, para que o professor possa fazer uma escolha acertada em relação às ferramentas computacionais e, em seguida, um planejamento coerente, outras questões estão envolvidas, como a formação desse profissional. Considera-se que, além da formação específica para o ensino de Estatística, faz-se primordial uma formação para o uso das tecnologias no processo educativo (SOUZA; LOPES, 2011).

Nessa categoria é apresentado o panorama das pesquisas levantadas que tratam da formação continuada de professores, sendo que, nesses moldes, enquadram-se apenas duas pesquisas: Amarante (2011) e Dias (2016), pois tais pesquisas focam suas propostas com professores já atuantes nos anos iniciais do Ensino Fundamental (AMARANTE, 2011; DIAS, 2016).

A pesquisa de Amarante (2011) foi realizada com professores de escolas rurais, que tinham como uma de suas características fundamentais o pouco uso do computador e, além disso, a não incorporação de ferramentas tecnológicas em suas aulas. No decorrer do relato de sua pesquisa, a autora menciona que nenhuma das escolas participantes possuía computadores (AMARANTE, 2011).

Em seu trabalho, é possível perceber a preocupação com uma formação pertinente do professor, para que suas dificuldades em relação aos conceitos estatísticos e ao uso dos recursos tecnológicos sejam, ao menos, minimizadas. Amarante (2011, p. 24), reconhecendo as contribuições das ferramentas computacionais no trabalho em sala de aula, acredita que empregar todo o potencial da tecnologia a serviço do aprimoramento do processo educativo é um grande desafio que se põe ao professor, considerando que é "o professor quem vai propor o uso de ferramentas informatizadas capazes de criar as situações favoráveis à aprendizagem dos conceitos e à superação das dificuldades dos alunos"(AMARANTE, 2011, p. 24).

A pesquisadora alerta para a necessidade de estudos futuros a respeito da utilização do TinkerPlots na formação de professores, nos quais sejam construídos os conceitos estatísticos, concedendo liberdade de manipulação e construção de diversas representações. "A investigação no âmbito da formação de professores poderá contribuir no seu próprio trabalho com o Tratamento da Informação em sala de aula"(AMARANTE, 2011, p. 149-150).

Dias (2016) vai além da simples formação para uso de um recurso tecnológico pronto para o ensino de Estatística, e propõe o desenvolvimento conjunto de um ambiente virtual que auxilie, tanto alunos, como professores dos anos iniciais. Em sua pesquisa, são nítidas as dificuldades encontradas em relação aos próprios conceitos estatísticos e à familiaridade com a 
tecnologia. A autora revela a necessidade de processos formativos que tratem dos conteúdos, do currículo e das tecnologias. Dias ressalta que:

[...] orientações e direcionamentos precisam ser dispendidos para que o docente compreenda a importância da Educação Estatística na vida cotidiana dos alunos e de si próprio, para que, ao longo de suas reflexões e de seu trabalho, possa mediar o conhecimento a respeito do tema (DIAS, 2016, p. 132).

Assim, observa-se que necessidade da formação continuada dos professores dos anos iniciais do Ensino Fundamental é evidenciada nas pesquisas de Amarante (2011) e Dias (2016). As pesquisadoras acreditam que possibilitar essa formação contribuirá para que as práticas em sala de aula possam ser aperfeiçoadas. Nesse sentido, destaca-se, a importância de novos estudos que envolvam, simultaneamente, o Ensino de Estatística, os recursos tecnológicos e a formação de professores (AMARANTE, 2011; DIAS, 2016).

\section{CONSIDERAÇÕES FINAIS}

As produções analisadas apresentam aspectos relevantes no ensino de Probabilidade e Estatística, explorando aplicativos de software específicos para o ensino com crianças da faixa etária que compreende os anos iniciais do Ensino Fundamental.

Um fato que chama a atenção, ao final desta análise, são as poucas produções nos mestrados profissionais, da área pesquisada, tanto em relação ao ensino de Estatística para os anos iniciais (6 dissertações), como para essa abordagem vinculada aos recursos tecnológicos (2 produções). Considerando as características dos mestrados profissionais em Ensino, pressupõe-se que mais pesquisas precisam ser realizadas com o intuito de contribuir com a prática em sala de aula e com o aprimoramento dos produtos e recursos que auxiliem no ensino de Estatística.

Observou-se que a maior parte dos aplicativos de software utilizado são comerciais, como é o caso TinkerPlots e do Tabletop. Acredita-se que essa característica possa inviabilizar o trabalho em algumas escolas.

Os conceitos trabalhados evidenciam um aspecto importante no ensino da Estatística, pois há uma ênfase maior nas questões de manipulação, leitura e análise dos dados e no entendimento desses processos, estimulando-se o raciocínio em detrimento do enfoque nos cálculos e nos procedimentos algébricos. Essa pode ser considerada a grande vantagem dos recursos tecnológicos para o ensino de Estatística. Esse aspecto é fundamental para que reflexões mais aprofundadas a respeito desse uso sejam desencadeadas. Os resultados positivos conseguidos nos estudos realizados demonstram que esse é um campo de pesquisa que necessita de avanços.

No que se refere às perspectivas para a formação de professores, evidencia-se que há necessidade de mais aprofundamento nessa área, já que apenas duas produções são desenvolvidas com professores. As pesquisas apresentadas demonstram resultados positivos na exploração dos conceitos estatísticos com os alunos por meio da tecnologia. Deve-se, agora, partir para a formação de professores para que, cada vez mais, esses recursos possam ser utilizados, contri- 
Ensino de Probabilidade e Estatística e Tecnologias da Informação e Comunicação: um cenário das produções brasileiras para os anos iniciais do Ensino Fundamental

buindo com abordagens mais motivadoras e coerentes no Tratamento da Informação. 


\section{REFERÊNCIAS}

ALVES, IM. A interpretação de gráficos em um ambiente computacional por alunos de escolas rurais. 2011. 165 f. 2011. Tese (Doutorado) - Dissertação (Mestrado em Educação Matemática e Tecnológica)-Centro de Educação, Universidade Federal de Pernambuco. Recife.

AMARANTE, Andreika Asseker. O uso do TinkerPlots para a exploração de dados por professores de escolas rurais. 2011. 156 f. 2011. Tese (Doutorado) - Dissertação (Mestrado em Educação Matemática e Tecnológica)-Centro de Educação, Universidade Federal de Pernambuco. Recife.

BATANERO, Carmen. Didáctica de la estadística. Granada: Universidad de Granada, 2001.

BATANERO, Carmen; DÍAZ, Carmen. Estadística con proyectos. Granada: Universidad de Granada, 2013.

BEN-ZVI, Dani. Statistical reasoning learning environment. Em Teial Revista de Educação Matemática e Tecnológica Iberoamericana-ISSN: 2177-9309, v. 2, n. 2, 2011.

BORBA, Marcelo de Carvalho; PENTEADO, Mirian Godoy. Informática e educação matemática. Belo Horizonte: Autêntica, 2012.

BRASIL. Parâmetros Curriculares Nacionais: Matemática, $1^{\circ}$ e $2^{\circ}$ ciclos do ensino fundamental. Ministério da Educação. Secretaria do Ensino Fundamental. Brasília, DF, 1997.

CAMPÊLO, Siquele Roseane de Carvalho. Software educativo Tinkerplots 2.0: possibilidades e limites para a interpretação de gráficos por estudantes do ensino fundamental. 2014 - 167 f. Dissertação (Mestrado em Educação Matemática e Tecnológica)- Centro de Educação, Universidade Federal de Pernambuco. Recife, 2014.

CARVALHO, Carolina. Interações entre pares: contributos para a promoção do desenvolvimento lógico e do desenvolvimento estatístico, no $\mathbf{7}^{\circ}$ ano de escolaridade. $2001-629$ f. Tese (Doutorado em Educação) - Faculdade de Ciências, Universidade de Lisboa. Lisboa, Portugal, 2001.

CAZORLA, Irene Mauricio; CASTRO, Franciana Carneiro de. O papel da estatística na leitura do mundo: o letramento estatístico. Publicatio UEPG: Ciências Sociais Aplicadas, v. 16, n. 1, p. 45-53, 2008. Disponível em: <http://www.revistas2.uepg.br/index.php/humanas/article/ view/617/605> Acesso em: 10 maio 2015.

CURCIO, Frances R. The effect of prior knowledge, reading and mathematics achievement, and sex on comprehending mathematical relationships expressed in graphs. final report. ERIC, 1981. Disponível em: <https://eric.ed.gov/?id=ED210185>. Acesso em: 23 maio 2016.

DIAS, Cristiane de Fatima Budek. Ambiente virtual de aprendizagem para o ensino de probabilidade e estatística nos anos iniciais do ensino fundamental. 2016 - Dissertação (Mestrado em Ensino de Ciência e Tecnologia)-Universidade Tecnológica Federal do Paraná. Ponta Grossa, 2016.

ESTEVAM, Everton José Goldoni; KALINKE, Marco Aurélio. Recursos tecnológicos e ensino de estatística na educação básica: um cenário de pesquisas brasileiras. Revista Brasileira de Informática na Educação, v. 21, n. 2, p. 104, 2013. Disponível em: <http://www.br-ie.org/ pub/index.php/rbie/article/view/2340>. Acesso em: 10 maio 2015. 
EUGÊNIO, R. Explorações sobre a média no TinkerPlots 2.0 por estudantes do ensino fundamental. 2013. 231 f. 2013 - Dissertação (Mestrado em Educação Matemática e Tecnológica)-Centro de Educação, Universidade Federal de Pernambuco. Recife.

GRANDO, Regina Célia; NACARATO, Adair Mendes; LOPES, Celi Espasandin. Narrativa de aula de uma professora sobre a investigação estatística. Educação \& Realidade, Universidade Federal do Rio Grande do Sul, v. 39, n. 4, 2014. Disponível em: <http://www.seer.ufrgs.br/ index.php/educacaoerealidade/article/view/45897>. Acesso em: 24 abr. 2015.

LIMA, Rosana Catarina Rodrigues de. Introduzindo o conceito de média aritmética na $4^{\mathrm{a}}$ série do ensino fundamental, usando o ambiente computacional. Pontifícia Universidade Católica de São Paulo, 2005. Disponível em: <http://www.sapientia.pucsp.br/tde_busca/arquivo.php? codArquivo=4507>. Acesso em 05 jun. 2015.

LIRA, Olga Cristina Teixeira; MONTEIRO, Carlos Eduardo F. Uso do computador na construção e interpretação de gráficos nos anos iniciais do ensino fundamental. II Simpósio Internacional de Educação Matemática-SIPEMAT, 2008. Disponível em: <http://www.gente.eti.br/ lematec/CDS/SIPEMAT08/?info_type=poster\&lang_user=en\&theme=theme3>. Acesso em: 9 maio 2016.

LIRA, Olga Cristina Teixeira; MONTEIRO, Carlos Eduardo F. Interpretação de dados a partir da utilização de ferramentas do software tinkerplots. Bolema, Rio Claro, v. 24, n. 40, p. $765-$ 788, 2011. Disponível em: <http://www.periodicos.rc.biblioteca.unesp.br/index.php/bolema/ article/view/5293>. Acesso em: 9 maio 2016.

LOPES, Celi Aparecida Espasandin. A probabilidade e a estatística no ensino fundamental: uma análise curricular. 1998 - Dissertação (Mestrado em Educação)-Faculdade de Educação, Universidade Estadual de Campinas, 1998. Disponível em: <http://www.bibliotecadigital. unicamp.br/document/?code=vtls000133638> . Acesso em 24 abr. 2015.

LOPES, Celi Espasandin. O ensino da estatística e da probabilidade na educação básica e a formação dos professores. Cad. Cedes, Campinas, v. 28, n. 74, p. 57-73, 2008. Disponível em: <http://www.scielo.br/scielo.php?script=sci_abstract\&pid=S010132622008000100005\& $\operatorname{lng}=\mathrm{en} \& \mathrm{nrm}=\mathrm{iso} \& \mathrm{t} \operatorname{lng}=\mathrm{pt}>$. Acesso em: 4 jun. 2015.

MARTINS, Maria Niedja Pereira. Professores desenvolvendo compreensões sobre amostragem com o software tinkerplots. In: XVII ENCONTRO NACIONAL DE ESTUDANTES DE PÓS-GRADUAÇÃO EM EDUCAÇÃO MATEMÁTICA, 2013. Anais... Vitória, 2013. Disponível em: <ftp://ftp.ifes.edu.br/cursos/Matematica/EBRAPEM/GDs/GD13/Sessao2/Sala_B3/ 1196-1879-1-PB.pdf>. Acesso em: 04 maio 2015.

MORAES, Luciana Rockenbach de. Como são propostas pesquisas em livros didáticos de ciências e matemática dos anos iniciais do ensino fundamental. 2011 - 75 f. Dissertação (Mestrado em Ensino Científico e Tecnológico)-Departamento de Ciências Exatas e da Terra, Universidade Regional Integrada do Alto Uruguai e das Missões, 2011. Disponível em: <http: //www.urisan.tche.br/admin/upload/luciana.pdf>. Acesso em 05 jun. 2015.

PONTE, João Pedro da; FONSECA, Helena. Orientações curriculares para o ensino da estatística: Análise comparativa de três países. Quadrante, Associação dos Professores de Matemática, p. 93-115, 2001.

SANTOS, Raquel; PONTE, João Pedro. O desenvolvimento de investigações estatísticas: Um estudo com futuros professores e educadores. Probabilidad Condicionada: Revista de didáctica de la Estadística, Universidad de Granada, n. 1, p. 455-462, 2013. 
SILVA, Edilza Maria da Conceição. Como são propostas pesquisas em livros didáticos de ciências e matemática dos anos iniciais do ensino fundamental. 2013 - Dissertação (Mestrado em Educação Matemática e Tecnológica)-Centro de Educação, Universidade Federal de Pernambuco. Recife. [s.d.].

SOUZA, Leandro de Oliveira; LOPES, Celi Espasandin. O uso de simuladores e a tecnologia no ensino da estocástica. Boletim de Educação Matemática, Universidade Estadual Paulista Júlio de Mesquita Filho, v. 24, n. 40, 2011. Disponível em: <http://www.periodicos.rc.biblioteca. unesp.br/index.php/bolema/article/view/5287>. Acesso em: 9 maio 2015.

STRAUSS, Sidney; BICHLER, Efraim. The development of children's concepts of the arithmetic average. Journal for Research in Mathematics Education, JSTOR, p. 64-80, 1988. Disponível em: <http://www.jstor.org/stable/749111?seq=1\&cid=pdf-reference\#references_tab_ contents>. Acesso em: 09 maio 2015.

VERGNAUD, Gérard. A classification of cognitive tasks and operations of thought involved in addition and subtraction problems. Addition and subtraction: A cognitive perspective, Lawrence Erlbaum Associate, p. 39-59, 1982.

VIALI, Lorí; SILVA, Mercedes Matte da. Sobre a necessidade de se iniciar o ensino/aprendizagem da estatística e da probabilidade na infância. Revista de Educação Matemática e Tecnológica Iberoamearicana, 2016. Disponível em: <http://www.repositorios.ufpe.br/revistas/ emteia/article/view/3891>. Acesso em: 9 dez. 2016. 